\title{
COMPOSIÇÃO FLORÍSTICA E ESTRUTURA DA REGENERAÇÃO NATURAL DE FLORESTA SECUNDÁRIA DE VÁRZEA BAIXA NO ESTUÁRIO AMAZÔNICO ${ }^{1}$
}

\begin{abstract}
João Ricardo Vasconcellos Gama ${ }^{2}$, Soraya Alvarenga Botelho ${ }^{3}$ e Michelliny de Matos Bentes-Gama ${ }^{4}$
RESUMO - O objetivo do presente estudo foi analisar a estrutura da regeneração natural de uma floresta explorada de várzea baixa localizada no município de Afuá, no norte do Estado do Pará. Foram amostradas 25 subparcelas de $100 \mathrm{~m}^{2}$, nas quais foram medidos a altura de todos os indivíduos das espécies arborescentes com altura (h) $\geq 0,30 \mathrm{~m}$ e diâmetro a 1,30 m do nível do solo (DAP) $<15,0 \mathrm{~cm}$ e o diâmetro das espécies com $\mathrm{h} \geq 3,0 \mathrm{~m}$ e DAP $<15,0 \mathrm{~cm}$, além de ter sido realizada a identificação botânica. Foram amostrados 13.380 indivíduos/ha, distribuídos em 63 espécies, 51 gêneros e 23 famílias botânicas. As espécies mais importantes da fitocenose foram: Euterpe oleracea, Astrocaryum murumuru, Crudia bracteata, Gustavia augusta e Inga edulis. Mais de $60 \%$ das espécies estudadas apresentaram padrão de distribuição agregado, e o índice de diversidade de Shannon-Weaver (H') foi de 3,05.
\end{abstract}

Palavras-chave: Fitossociologia, floresta de várzea e Amazônia.

\section{FLORISTIC COMPOSITION AND NATURAL REGENERATION OF A SECONDARY LOW FLOODPLAIN FOREST IN THE AMAZONIAN ESTUARY}

\begin{abstract}
This paper aimed to analyze the natural regeneration structure of an exploited low floodplain forest located at EMAPA forestlands, Afuá County, in northern Pará. Sampling was accomplished in 25 sub-plots of $100 \mathrm{~m}^{2}$. In each sub-plot of $100 \mathrm{~m}^{2}$, all individuals with height $(h) \geq 0.30 \mathrm{~m}$ and diameter at $1,30 \mathrm{~m}$ above ground level $(D B H) \leq 15.0 \mathrm{~cm}$ were surveyed and were botanically identified, as well as all the plants with $(h) \geq 3.0 \mathrm{~m}$ and diameter at $1.30 \mathrm{~m}$ above ground level $(\mathrm{DBH})<15.0 \mathrm{~cm}$. A total of 13.380 individuals/ha distributed in 63 species, 51 genera and 23 botanical families were sampled. The most important species of the phytocenosis were: Euterpe oleracea, Astrocaryum murumuru, Crudia bracteata, Gustavia augusta and Inga edulis. Over $60 \%$ of the species studied in the area showed a clustering distribution pattern and a Shannon-Weaver index ( $\left.H^{\prime}\right)$ of 3.05.
\end{abstract}

Key words: $\quad$ Phytosociology, floodplain forest and Amazonia.

\section{INTRODUÇÃO}

A regeneração natural decorre da interação de processos naturais de restabelecimento do ecossistema florestal. É, portanto, parte do ciclo de crescimento da floresta e refere-se às fases iniciais de seu estabelecimento e desenvolvimento. O estudo da regeneração natural permite a realização de previsões sobre o comportamento e desenvolvimento futuro da floresta, pois fornece a relação e a quantidade de espécies que constituem o seu estoque, bem como suas dimensões e distribuição na área (Carvalho, 1982). Com estas informações, o silvicultor

\footnotetext{
1 Recebido para publicação em 3.5.2001.
}

Aceito para publicação em 6.11.2002.

2 Engenheiro Florestal, M.S., Doutorando do Departamento de Engenharia Florestal da Universidade Federal de Viçosa - UFV, 36571-000 Viçosa-MG, <jrvgama@vicosa.ufv.br>; ${ }^{3}$ Professora do Departamento de Ciências Florestais, Universidade Federal de Lavras, Caixa Postal 37, 37200-000 Lavras-MG , <sbotelho@ufla.br>. ${ }^{4}$ Engenheira Florestal, M.S., Pesquisadora da Embrapa Rondônia, BR 364, km 5,5, Caixa Postal 406, 78900-970 Porto Velho-RO, <mbgama@cpafro.embrapa.br>. 
pode incentivar o crescimento e maximizar o volume das espécies desejáveis por unidade de área.

Através do estudo da regeneração natural são obtidas informações sobre autoecologia, estádio sucessional, efeitos da exploração florestal, entre outras informações importantes que norteiam as intervenções silviculturais previstas nos planos de manejo (Higuchi et al., 1985).

Hoje em dia, os estudos a respeito da estrutura da regeneração natural nos ecossistemas amazônicos se restringem basicamente às formações florestais de terrafirme. Em se tratando de florestas de várzea, poucos estudos foram realizados, dentre os quais estão os de Oliveira (1992), Higuchi et al. (1994) e Macedo (1996).

Nesse sentido, os objetivos do presente estudo foram conhecer a composição florística e analisar a estrutura e a distribuição espacial da regeneração natural em uma floresta de várzea baixa, na região de estuário amazônico no Estado do Pará.

\section{MATERIAL E MÉTODOS}

\section{1. Área de Estudo}

A área de estudo está localizada na propriedade florestal da Exportadora de Madeiras do Pará Ltda. EMAPA, no município de Afuá, Estado do Pará. A área total é de 1.200 ha, dos quais 80 ha são de floresta de várzea baixa que sofreu exploração madeireira no período de 1955 até fevereiro de 1992. Os valores médios anuais de precipitação e temperatura correspondem, respectivamente, a $2.500 \mathrm{~mm}$ e $26^{\circ} \mathrm{C}$ (SUDAM, 1984). O solo é do tipo hidromórfico gleizado pouco húmico VIEIRA (1988).

\subsection{Amostragem e Coleta dos Dados}

Foi delimitado nos 80 ha de floresta de várzea baixa um talhão de 60 ha $(600 \times 1.000$ m), onde se distribuíram sistematicamente 25 parcelas de $20 \times 250 \mathrm{~m}$ (0,5 ha), que continham uma subparcela de 10 x 10 m para o inventário da regeneração natural. Nas subparcelas foram mensurados todos os indivíduos das espécies arborescentes (arbóreas e palmeiras) com altura $(\mathrm{h}) \geq 0,3 \mathrm{~m}$ e diâmetro a 1,30 $\mathrm{m}$ de altura do solo (DAP) $<15,0 \mathrm{~cm}$, dos quais foram anotadas as seguintes informações: nome regional; diâmetro dos indivíduos com $\mathrm{h} \geq 3,0 \mathrm{~m}$ a DAP $<15 \mathrm{~cm}$; altura total; e qualidade da regeneração natural: viva em pé, viva caída e com danos.

R. Árvore, Viçosa-MG, v.26, n.5, p.559-566, 2002
As espécies também foram classificadas, segundo a sua categoria de uso, em: comerciais - comercializadas no mercado nacional e internacional; potenciais - comercializadas no mercado local e regional; e não-comerciais. Todas as espécies amostradas tiveram seu material botânico coletado e passaram pelos processos de herborização. Posteriormente, foram identificadas quanto à família, ao gênero e à espécie, por especialistas do Laboratório de Botânica da Embrapa-CPATU, em Belém-PA. Os dados foram coletados no período de dezembro de 1998 a fevereiro de 1999. Adotou-se o sistema de classificação de Cronquist (1988).

\subsection{Análise dos Dados}

Foi realizada a definição do grupo ecológico a que pertencia cada espécie inventariada, considerando-se a proposta de Oliveira-Filho (1994), juntamente com revisões bibliográficas e observações de campo, adotando-se as seguintes categorias: pioneira, clímax exigente de luz e clímax tolerante à sombra.

A suficiência amostral foi definida através do procedimento REGRELRP - Regressão Linear com Resposta em Platô, do Sistema para Análises Estatísticas - SAEG v. 5.0 da Universidade Federal de Viçosa (Ferreira, 1988; Volpato, 1994; Nappo, 1999).

Para análise da estrutura horizontal da regeneração natural os parâmetros considerados foram: densidade, freqüência, dominância e índice de valor de importância, os quais estão descritos em Curtis \& Mcintosh (1951).

No estudo da estrutura vertical da regeneração natural foram utilizadas as classes de tamanho (CT) sugeridas pelaFAO (1971), sendo:classeCT 1: 0,3 m $\leq \mathrm{h}<1,5 \mathrm{~m}$; CT2: $1,5 \mathrm{~m} \leq \mathrm{h}<3,0 \mathrm{~m}$; CT3: $\mathrm{h} \geq 3,0 \mathrm{~m}$ e DAP $<5,0 \mathrm{~cm}$; CT4: 5,0 cm $\leq$ DAP < 10,0 cm; e CT5: 10,0 cm $\leq$ DAP $<15,0 \mathrm{~cm}$. As fórmulas empregadas para estimativa das classes absoluta e relativa de tamanho da regeneração natural foram propostas por Finol (1971). Outro parâmetro também estimado foi o valor de importância ampliado para a regeneração natural (IVIARN), obtido pela expressão:

$$
\operatorname{IVIARN}_{i}=\operatorname{IVIRN_{i}}+C R T N_{i}
$$

em que $I V I R N_{i}=$ índice de valor de importância para a i-ésima espécie; e $C R T R N_{i}=$ classe relativa de tamanho da regeneração natural para a i-ésima espécie. 
Para cálculo da diversidade foi empregado o índice de Shannon-Weaver, e o padrão de distribuição espacial foi estimado através do índice de Morisita (Ludwige \& Reynolds, 1988). Para cálculo do índice de Morisita foram consideradas apenas as espécies que ocorreram em pelo menos duas parcelas.

\section{RESULTADOS E DISCUSSÃO}

\subsection{Composição Florística}

Através do inventário da regeneração natural das espécies arborescentes foram registrados 63 espécies, 51 gêneros e 23 famílias botânicas (Quadro 1). Dentre as famílias com maior riqueza de espécie encontraram-se: Leguminosae (18), Arecaceae (6) e Chrysobalanaceae (5). A ocorrência destas famílias com o maior número de espécies é compatível com o encontrado em floresta de várzea do estuário amazônico por Macedo (1996).

A categorização das espécies entre os grupos ecológicos resultou em $9,5 \%$ de pioneiras, $42,9 \%$ de clímax exigentes de luz e 42,9\% de clímax tolerantes à sombra, enquanto 4,8\% não foram classificadas. Relacionandose os grupos ecológicos com as categorias de uso das espécies, foram constatadas entre as espécies comerciais e potenciais duas pioneiras, nove clímax exigentes de luz e seis clímax tolerantes à sombra. Com relação às não-comerciais, ocorreram 4 pioneiras, 18 clímax exigentes de luz e 21 clímax tolerantes à sombra. Verificou-se que após 7 anos da última exploração a floresta apresenta boa recuperação, tendo em vista a ocorrência de um baixo número de espécies pioneiras no sub-bosque (Quadro 1).

\subsection{Suficiência Amostral}

Através da análise de regressão com resposta em platô verificou-se que 14 parcelas seriam suficientes para representar a composição florística do nível de inclusão estudado (Figura 1).

\subsection{Análise da Estrutura da Regeneração Natural}

Foram encontrados 13.380 indivíduos/ha, devendo ser ressaltado que a área basal para os indivíduos contidos no intervalo de $\mathrm{h} \geq 3,0 \mathrm{~m}$ a DAP $<15,0 \mathrm{~cm}$ foi de $11,13 \mathrm{~m}^{2} /$ ha. A regeneração apresentou altura média de 2,0 m e máxima de $13 \mathrm{~m}$. A distribuição do número de indivíduos por classe de altura demonstrou a tendência esperada de diminuição do número de plantas com o aumento das classes de tamanho (Figura 2).

As espécies que ocorreram na maior classe de altura ( $\mathrm{h} \geq 12 \mathrm{~m}$ ) foram Euterpe oleracea, Tachigali myrmecophila, Inga edulis, Eschweilera coriacea, Licania canescens, Licania heteromorpha, Licania macrophylla, Hevea brasiliensis e Pentaclethra macroloba. Conforme Bentes-Gama (2000), todas estas espécies ocorreram no estrato superior da floresta de várzea baixa estudada.

A Figura 3 mostra que as classes de tamanho de regeneração natural apresentaram distribuição em 'J' invertido apenas para o porcentual de indivíduos. Já com relação ao número de espécies esta tendência não ocorreu. Segundo a FAO (1971) e Carvalho (1982), isso se deve ao fato de a floresta ainda encontrar-se em fase de construção.

As espécies mais importantes da área de estudo foram Euterpe oleracea, Astrocaryum murumuru, Crudia bracteata, Gustavia augusta e Inga edulis (Quadro 1). Analisando o potencial econômico e ecológico das espécies que apresentaram IVIARN $\geq 10,0$, foi possível verificar entre as comerciais: Euterpe oleracea e Virola surinamensis, que são as espécies de maior demanda no estuário amazônico. De Euterpe oleracea se retira o palmito de açaí, que é o produto não-madeireiro mais comercializado em área de várzea. Virola surinamensis tem sua madeira preferencialmente aproveitada por serrarias e indústrias de compensado localizadas nessa área.

Quanto às espécies potenciais, as mais importantes foram Symphonia globulifera e Caraipa grandiflora, que são espécies utilizadas em pequenas serrarias para o desdobro de tábuas. Em relação às espécies não-comerciais, as principais foram Astrocaryum murumuru, Miconia ceramicarpa, Inga edulis e Licania canescens, cujos frutos são consumidos pela fauna local. Outras nãocomerciais de grande aproveitamento são: Crudia bracteata, que é utilizada para confeccionar varas que servem para empurrar pequenas embarcações; Gustavia augusta, cuja madeira é utilizada em construções rústicas, na fabricação de cabos de machado e ainda tem seus frutos consumidos por animais silvestres; Zygia juruana, que tem sua madeira muito utilizada como lenha; e Eschweilera coriacea, cuja casca é utilizada na fabricação de cordas.

R. Árvore, Viçosa-MG, v.26, n. 5, p.559-566, 2002 
Quadro 1 - Relação das espécies arborescentes em ordem decrescente de importância amostradas em várzea baixa, na propriedade florestal da EMAPA, município de Afuá, Pará, em que grupo de uso (GU) - comercial (C), potencial (P) e não-comercial (NC); grupo ecológico (GE) - pioneira (PI), clímax exigente de luz (CL) e clímax tolerante à sombra (CS); padrão de distribuição espacial da espécie (PDE) - agregado (AG) e aleatório (AL); freqüência relativa (FRRN); densidade relativa (DRRN); dominância relativa (DoRRN); índice de valor de importância (IVIRN); classe de tamanho (CT) - plantas/ha; classe relativa de tamanho (CRT); e índice de valor de importância ampliado (IVIARN)

Table 1 - List of tree species in decreasing order of importance of species sampled in a low floodplain forest, at EMAPA forestlands, Afuá, Pará. Where: Category of use $(G U)$ - commercial (C), potential (P), and non-commercial (NC); ecological species group (GE) - pioneer (PI), light demanding (CL) and shade tolerant (CS); spatial distribution pattern $(P D E)$ - clustering $(A G)$ and randomized $(A L)$; relative frequency (FRRN); relative density (DRRN); relative dominance $(D o R R N)$; importance value index (IVIRN); class size (CT) - plants/ha; relative class size (CRT); and amplified value of importance index (IVIARN)

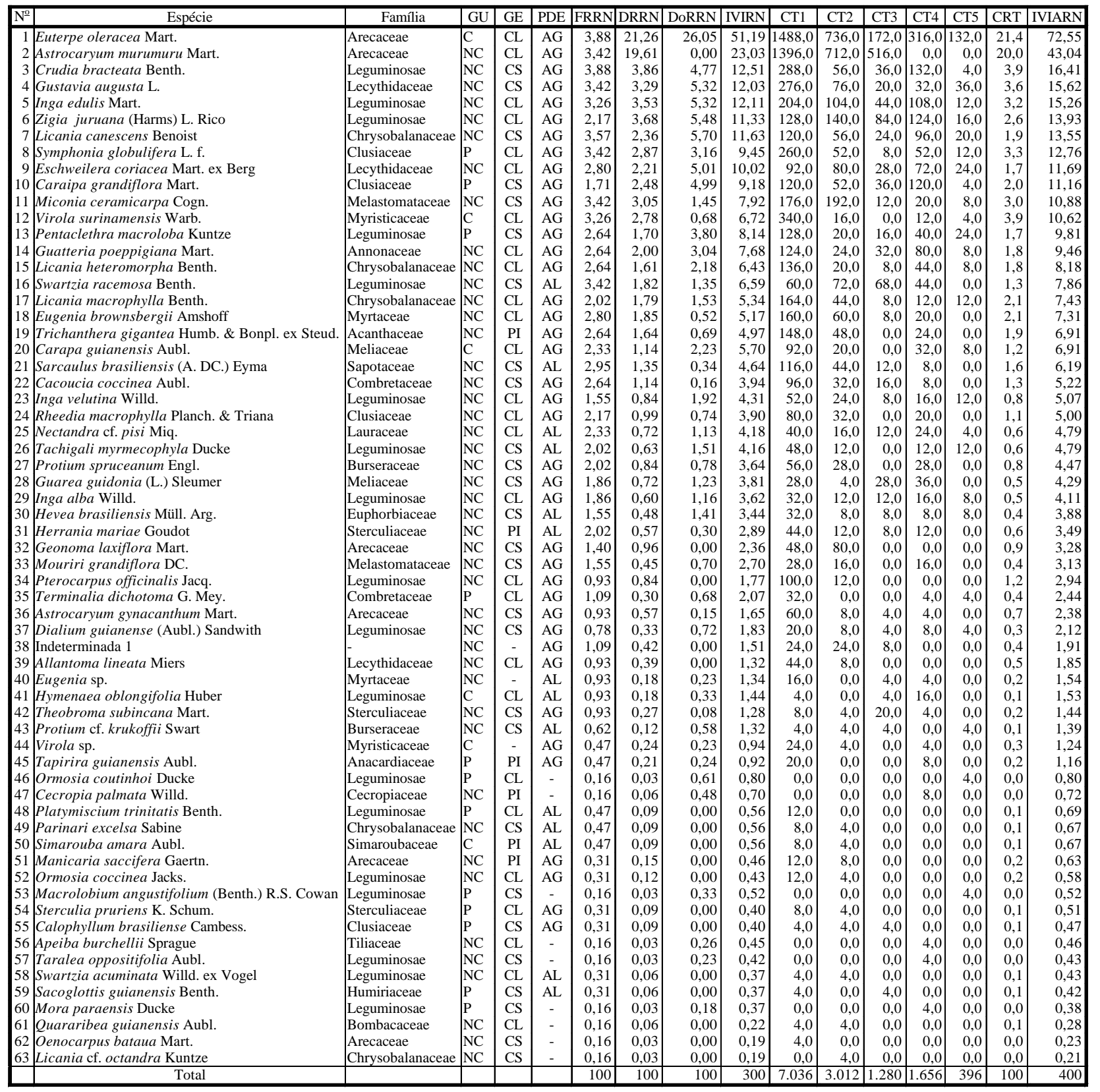




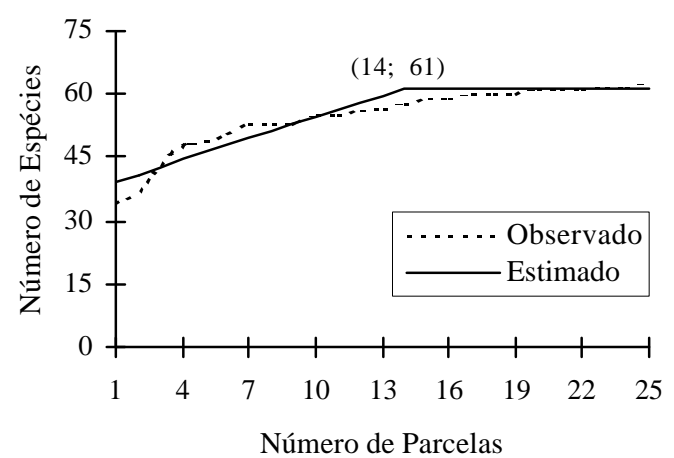

Figura 1 - Suficiência amostral para a regeneração natural de várzea baixa. Propriedade florestal da EMAPA, município de Afuá, Pará.

Figure 1 - Sampling sufficiency for the natural regeneration of a low floodplain forest. EMAPA forestlands, Afuá, Pará.

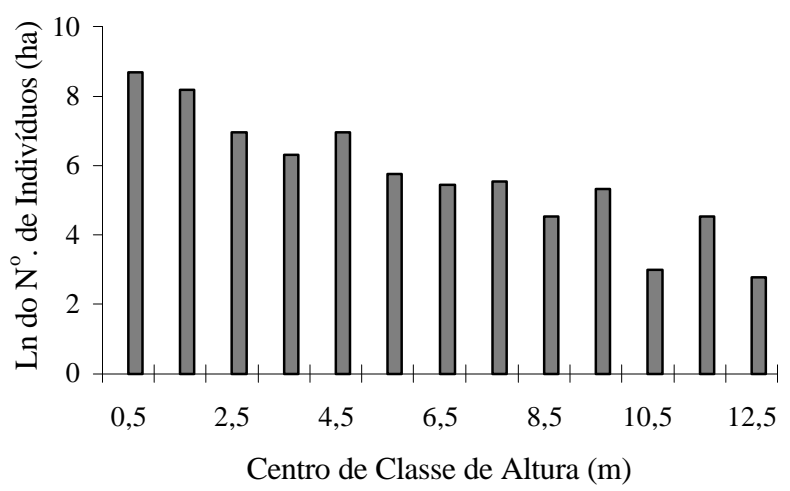

Figura 2 - Distribuição logaritmizada do número de indivíduos por classe de altura. Propriedade florestal da EMAPA, município de Afuá, Pará.

Figure 2 - Logarithmic distribution of the number of individuals per class of height. EMAPA forestlands, Afuá, Pará.

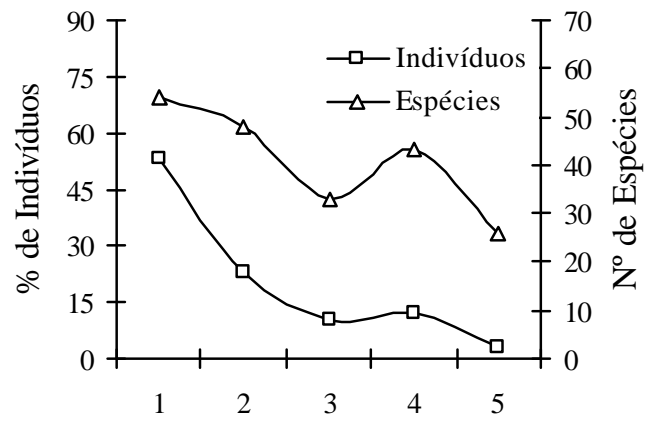

Classe de Tamanho

Figura 3 - Número de espécies e porcentual de indivíduos em relação às classes de tamanho utilizadas. Propriedade florestal da EMAPA, município de Afuá, Pará.

Figure 3- Number of species and individual percent in relation to the class sizes used. EMAPA forestlands, Afuá, Pará.
Além das espécies já citadas, ressaltam-se outras que ocorreram em todas as classes de tamanho, bem distribuídas na área de estudo e que são importantes para manutenção da fauna e subsistência dos ribeirinhos, a saber: Hevea brasiliensis (frutos consumidos por animais silvestres e utilizados para confecção de artesanato); Licania macrophylla (frutos consumidos por animais silvestres e casca do tronco utilizada na medicina caseira); Inga velutina e Inga alba (frutos comercializados em feiras livres e consumidos por animais silvestres); Licania heteromorpha e Guatteria poeppigiana (madeira roliça utilizada em construções rústicas); e Pentaclethra macroloba (fuste processado pelas pequenas serrarias para o desdobro de tábuas).

Entre as espécies citadas, poucas são comercializadas, embora a maioria seja utilizada para obtenção de produtos não-madeireiros e, ou, possa substituir as espécies que são muito exploradas. Há, portanto, a necessidade de efetuar estudos que confirmem suas propriedades tecnológicas e divulguem amplamente suas características.

Comparando-se os resultados de densidade, frequiência, dominância e distribuição dos indivíduos entre as classes de tamanho com os trabalhos de Oliveira (1992), Higuchi et al. (1994) e Macedo (1996), que estudaram florestas de várzea não-exploradas, é possível inferir que a exploração realizada colaborou para o melhor desenvolvimento da regeneração natural. Resultados similares foram encontrados por Pitt (1961), Silva (1989) e Veríssimo et al. (1996). Entretanto, Bentes-Gama (2000) relatou que o período de sete anos após a última exploração ainda não foi o bastante para que a floresta apresentasse um estoque de exploração (DAP $\geq 45 \mathrm{~cm}$ ) suficiente para suportar a exploração que é realizada no estuário amazônico, que corresponde a extração de pelo menos oito árvores comerciais/ha.

Quanto à tendência de distribuição espacial das espécies (Quadro 1), verificou-se que 63,5\% apresentaram padrão agregado e $22,2 \%$, aleatório. O padrão de distribuição espacial não foi calculado para 14,3\% das espécies, uma vez que elas só ocorreram em uma parcela. Entre as espécies mais importantes, todas apresentaram padrão de distribuição agregado, tendência esta também verificada por Barros (1986), Carvalho (1992) e Santana et al. (1997).

Como causas de agregação dos indivíduos podemse citar alguns fatores que ocorrem de maneira diferenciada no interior da floresta, como: temperatura, umidade,

R. Árvore, Viçosa-MG, v.26, n.5, p.559-566, 2002 
GAMA, J.R.V. et al.

disponibilidade de luz, topografia, fertilidade do solo etc. Yared (1996) comentou que o padrão de distribuição é afetado pela dispersão de sementes, que por sua vez é bastante dependente da fauna e da ocorrência de ventos. No caso da floresta estudada, supõe-se que a agregação dos indivíduos de grande parte das espécies esteja relacionada com a longa duração e alta intensidade de exploração, grande ocorrência de fontes de sementes, baixa freqüência de predadores, condições edafoclimáticas diferenciadas, diferentes níveis de inundação da floresta, entre outros fatores.

No contexto da exploração florestal uma das características benéficas da agregação de espécies refere-se à redução de custos por ocasião da execução das atividades de corte e extração. Entretanto, se esta exploração for realizada pelo modo convencional, ou seja, sem considerar as prescrições do sistema silvicultural, a sustentabilidade ecológica das espécies que apresentam este padrão de distribuição espacial pode ficar ameaçada.

\subsection{Parâmetros Qualitativos da Regeneração Natural}

Verificou-se que a qualidade da regeneração natural foi satisfatória (Quadro 2), pois mais de 95\% dos indivíduos apresentaram-se bem formados e eretos. O porcentual de indivíduos caídos (tombados) foi menor que $0,36 \%$, excetuando as espécies potenciais, que apresentaram $2,25 \%$ de seus indivíduos caídos.

Quadro 2 - Qualidade da regeneração natural de acordo com o potencial de utilização das espécies inventariadas na propriedade florestal da EMAPA, município de Afuá, Pará

Table 2 - Natural regeneration quality according to the use potential of the inventoried species at EMAPA forestlands, Afuá, Pará

\begin{tabular}{|l|c|c|c|}
\hline \multirow{2}{*}{ Grupo de Uso } & \multicolumn{3}{|c|}{ Qualidade da Regeneração Natural (\%) } \\
\cline { 2 - 4 } & Com danos & Viva caída & Viva em pé \\
\hline Comerciais & 0,47 & 0,35 & 99,19 \\
Potenciais & 2,62 & 2,25 & 95,13 \\
Não comerciais & 4,64 & 0,36 & 95,00 \\
\hline
\end{tabular}

Quanto aos indivíduos que apresentaram algum dano estrutural (quebrado, roído, cortado, entre outros), verificou-se que a maior incidência ocorreu entre as espécies não-comerciais $(4,64 \%)$, podendo-se destacar Sarcaulus brasiliensis, Eschweilera coriacea e Miconia ceramicarpa, que apresentaram a maior preferência pelos animais silvestres.

R. Árvore, Viçosa-MG, v.26, n.5, p.559-566, 2002

\section{5. Índice de Diversidade}

O índice de diversidade de Shannon-Weaver ( $\left.\mathrm{H}^{\prime}\right)$ foi de 3,05. Bentes-Gama (2000), analisando o estrato arbóreo (DAP $\geq 15 \mathrm{~cm}$ ) na mesma área e ambiente, encontrou maior diversidade $\left(\mathrm{H}^{\prime}=3,35\right)$, enquanto Macedo (1996) obteve H' = 2,76 para floresta de várzea nãoexplorada no estuário amazônico; já na floresta de várzea que sofreu redução de $75 \%$ de área basal o autor obteve $\mathrm{H}^{\prime}=2,87$ no segundo ano após a exploração, constando que mesmo uma exploração pesada pode aumentar a diversidade de espécies arborescentes da regeneração natural. Bentes \& Maciel (1994), inventariando a vegetação com DAP $\geq 10 \mathrm{~cm}$ em floresta de várzea no rio Cajuúna, encontraram $\mathrm{H}^{\prime}=2,56$, e no rio Urucu, $\mathrm{H}^{\prime}=3,17$, ambos os locais no município de Afuá-PA.

Observou-se que os valores desse índice variaram de 2,5 a 3,6 em florestas de várzea do estuário amazônico. Em floresta de terra-firme na Amazônia, Martins (1991) relatou que os valores de H' variam de 3,5 a 4,7. De modo geral, notou-se que a diversidade muda de acordo com os níveis de abordagem do inventário florestal. Portanto, recomenda-se que as comparações devem ser restritas às mesmas classes de tamanho, ou realizadas com bastante cautela. Rolim \& Nascimento (1997) ainda ressaltaram que o índice de diversidade de ShannonWeaver apresenta pequenas diferenças, entretanto significativas, entre diferentes intensidades amostrais.

\section{CONCLUSÃo}

Com base na análise e discussão dos resultados, pode-se concluir que: Euterpe oleracea, Astrocaryum murumuru, Crudia bracteata, Gustavia augusta, Inga edulis, Zygia juruana, Licania canescens, Symphonia globulifera, Eschweilera coriacea, Caraipa grandiflora, Miconia ceramicarpa e Virola surinamensis foram as espécies mais importantes da fitocenose; Hevea brasiliensis, Licania macrophylla, Inga velutina e Inga alba são espécies potenciais para fornecimento de produtos florestais não-madeireiros; mais de $60 \%$ das espécies apresentaram padrão de distribuição agregado; após o intervalo de sete anos sem exploração a floresta demonstrou boa recuperação estrutural, como também diversidade $\left(H^{\prime}=3,05\right)$; e a qualidade da regeneração natural foi satisfatória, pois menos de $5 \%$ dos indivíduos apresentaram algum dano estrutural. 


\section{REFERÊNCIAS BIBLIOGRÁFICAS}

BARROS, P. L. C. Estudo fitossociológico de uma floresta tropical úmida no planalto de Curuá-Una, Amazônia brasileira. Curitiba: UFPR, 1986. 147 p. Tese (Doutorado em Ciências Florestais) - Universidade Federal do Paraná, 1986.

BENTES, M. P. M.; MACIEL, U. N. Composição florística, estrutura e multipropósito de espécies arbóreas de mata de várzea no município de Afuá, arquipélago de Marajó, PA. Belém: MPEG/DBO, 1994. $30 \mathrm{p}$.

BENTES-GAMA, M. M. Estrutura, valoração e opções de manejo sustentado para uma floresta de várzea na Amazônia. Lavras: UFLA, 2000. 206 p. Dissertação (Mestrado em Engenharia Florestal) - Universidade Federal de Lavras, 2000.

CARVALHO, J. O. P. Análise estrutural da regeneração natural em floresta tropical densa na região do Tapajós no Estado do Pará. Curitiba: UFPR, 1982. 128 p. Dissertação (Mestrado em Ciências Florestais) Universidade Federal do Paraná, 1982.

CARVALHO, J. O. P. Structure and dynamics of a logged over Brazilian Amazonian rain forest. Oxford: University of Oxford, 1992. 215 p. Thesis (Doctorate in Forestry Science) - University of Oxford, 1992.

CRONQUIST, A. The evolution and classification of flowering plants. New York: The New York Botanical Garden, 1988. 555 p.

CURTIS, J. T.; McINTOSH, R. P. An upland forest continuum in the prairie forest border region of Wisconsin. Ecology, v. 32, p. 476-496, 1951.

FERREIRA, R. L. C. Análise estrutural da vegetação da Estação Florestal da Experimentação de Açú - RN, como subsídio básico para o manejo florestal. Viçosa: UFV, 1988. 91p. Dissertação (Mestrado em Ciências Florestais) - Universidade Federal de Viçosa, 1988.

FINOL U. H. Nuevos parametros a considerarse en el analisis estructural de las selvas virgines tropicales. Revista Forestal Venezolana, v. 14, n. 21, p. 29-42, 1971.

FOOD AND AGRICULTURE ORGANIZATION OF THE UNITED NATIONS - FAO. Silvicultural research in the Amazon. Roma: Food and Agriculture Organization of the United Nations, 1971. 192 p. (Technical Report, 3).
HIGUCHI, N. et al. Exploração florestal nas várzeas do Estado do Amazonas: seleção de árvores, derruba e transporte. In: UPDATING SEMINAR ON HARVESTING AND WOOD TRANSPORTATION, 8., 1994, Curitiba. Anais... Curitiba: UFPr, 1994. p. 168-193.

HIGUCHI, N. et al. Bacia 3 - Inventário diagnóstico da regeneração natural. Acta Amazonica, v. 15, n. 1/2, p. 199233, 1985.

LUDWIG, J. A.; REYNOLDS, J. F. Statistical ecology: a primer on methods and computing. New York: John Wiley \& Sons, 1988. $337 \mathrm{p}$.

MACEDO, D. S. M. S. Estrutura e manejo de uma floresta de várzea do estuário amazônico. Piracicaba: ESALQ, 1996. 117 p. Dissertação (Mestrado em Ciências Florestais) - Escola Superior de Agricultura "Luiz de Queiroz", 1996.

MARTINS, F. R. Estrutura de uma floresta mesófila. Campinas: UNICAMP, 1991. 246 p.

NAPPO, M. E. Inventário florístico e estrutural da regeneração natural no sub-bosque de povoamentos homogêneos de Mimosa scabrella Bentham implantados em áreas mineradas, em Poços de Caldas, Minas Gerais. Lavras: UFLA, 1999. 87 p. Dissertação (Mestrado em Engenharia Florestal) - Universidade Federal de Lavras, 1999.

OLIVEIRA, M. V. Exploração de madeira em várzea pelo método tradicional no paraná Abufari no médio rio Purus. Rio Branco: EMBRAPA-CPAF, 1992. 15 p. (Boletim de Pesquisa, 7).

OLIVEIRA-FILHO, A. T. Estudos ecológicos da vegetação como subsídio para programas de revegetação com espécies nativas: uma proposta metodológica. Cerne, v. 1, n. 1, p. 64-72, 1994.

PITT, J. Possible methods of regenerating and improving some of the Amazon forests. Caribbean Forester, v. 22, n. 1/2, p. 26-32, 1961.

ROLIM, S. G.; NASCIMENTO, H. E. M. Análise da riqueza, diversidade e relação espécie-abundância de uma comunidade arbórea tropical em diferentes intensidades amostrais. Scientia Forestalis, v. 52, p. 7-16, 1997.

SANTANA, J. A. S.; BARROS, L. P.; JARDIM, F. C. S. Análise da vegetação de regeneração natural na floresta tropical úmida em Paragominas-PA. Boletim FCAP, n. 28, p. 9-35, 1997.

R. Árvore, Viçosa-MG, v.26, n.5, p.559-566, 2002 
SILVA, J. N. M. The behaviour of the tropical rain forest of the Brazilian Amazon after logging. Oxford: University of Oxford, 1989. 302 p. Thesis (Doctorate in Forestry Science) - University of Oxford, 1989.

SUDAM/PROJETO DE HIDROLOGIA E CLIMATOLOGIA DA AMAZÔNIA. Atlas Climatológico da Amazônia Brasileira. Belém: SUDAM, 1984. 125 p.

VERÍSSIMO, A. et al. Impactos sociais, econômicos e ecológicos da exploração seletiva de madeiras numa região de fronteira na Amazônia oriental: o caso de Tailândia. In: BARROS, A. C.; VERÍSSIMO, A. (Eds.) A expansão da atividade madeireira na Amazônia: impactos e perspectivas para o desenvolvimento do setor florestal no Pará. Belém: IMAZON, 1996. 168 p.
VIEIRA, L. S. Manual da ciência do solo: com ênfase aos solos tropicais. 2.ed. São Paulo: Agronômica Ceres, 1988. $464 \mathrm{p}$.

VOLPATO, M. M. L. Regeneração natural em uma floresta secundária no domínio de mata Atlântica: uma análise fitossociológica. Viçosa: UFV, 1994. 123 p. Dissertação (Mestrado em Ciências Florestais) Universidade Federal de Viçosa, 1994.

YARED, J. A. G. Efeitos de sistemas silviculturais na florística e na estrutura de florestas secundárias e primárias na Amazônia Oriental. Viçosa: UFV, 1996. 179 p. Tese (Doutorado em Ciências Florestais) Universidade Federal de Viçosa, 1994. 